\title{
Quantification of Myocardial Perfusion Lesions using Spatially Variant Finite Mixture Modelling of DCE-MRI
}

\author{
Yalei Yang ${ }^{1}$, Hao Gao ${ }^{1}$, Colin Berry ${ }^{2}$, Aleksandra Radjenovic ${ }^{2, *}$, Dirk Husmeier ${ }^{1, *}$ \\ ${ }^{1}$ School of Mathematics \& Statistics, University of Glasgow \\ University Place Glasgow, G12 8QQ, United Kingdom \\ y.yang.2@research.gla.ac.uk; hao.gao@glasgow.ac.uk; dirk.husmeier@glasgow.ac.uk \\ ${ }^{2}$ Institute of Cardiovascular \& Medical Sciences, University of Glasgow \\ BHF Glasgow Cardiovascular Research Centre (GCRC), 126 University Place, Glasgow, G12 8TA, United Kingdom \\ colin.berry@glasgow.ac.uk; aleksandra.radjenovic@glasgow.ac.uk \\ ${ }^{*}$ Corresponding Author
}

\begin{abstract}
Dynamic Contract Enhanced Magnetic Resonance (MR) Imaging (DCE-MRI) can reveal differences in myocardial perfusion (microvascular or capillary blood flow) within the myocardium. The detection and quantification of hypo-perfused lesions within the myocardium is important for understanding aetiology of coronary heart disease (CHD). In this paper, a modification of a traditional method, the Expectation-Maximization (EM) algorithm for Gaussian Mixture Models (GMM), is implemented. This modification, the Spatially Variant Finite Mixture Model (SVFMM), is able to take the neighbourhood information of a voxel in the MR image into account. An experiment based on both synthetic and real images illustrates and quantifies the improvement achieved with SVFMM over the traditional GMM method.
\end{abstract}

Keywords: DCE-MRI, myocardial perfusion, lesion quantification, Gaussian Mixture Model, Spatially Variant Finite Mixture Model.

\section{Introduction}

Coronary heart disease (CHD) is the major cause of death all over the world[1]. It caused 7,249,000 deaths in 2008, which is $12.7 \%$ of total global mortality [1]. Myocardial ischaemia (inadequate perfusion or capillary blood flow) is implicated in many forms of CHD. This disease happens when the cardiac blood supply is impaired. In particular it happens mostly when one or more coronary arteries are narrowed. The limited supply of blood causes hypoxia (reduced oxygen supply) in the myocardium. However, it is challenging to accurately quantify the hypoxia in the myocardium. An effective and non-invasive method for the assessment of myocardial perfusion is dynamic contrast-enhanced Magnetic Resonance Imaging (DCE-MRI) [2]. This imaging method involves intravenous administration of MRI contrast agent that selectively alters signal intensity (SI) in proportion to the local tissue levels of microvascular perfusion, and dynamic, time-resolved capture of SI changes during the first pass of the contrast agent through the microvasculature.

DCE-MRI can be analysed using a variety of quantitative methods [2], but the mainstay of clinical practice is still visual (qualitative) observation of the presence of ischaemic lesions, which manifest themselves as areas of reduced SI during first pass of contrast agent (in T1-weighted DCE-MRI sequences, such as those used in our study). Another approach involves computation of synthetic parametric maps [2]: spatial representations of various perfusion-related indices. This approach has a potential advantage of detecting, delineating and quantifying areas of hypo-perfusion that are below the visual threshold, due to low intensity of pathological change and/or low Signal-to-noise ratio (SNR) of the source images.

The aim of our work is to design methods that will allow delineation and quantification of myocardial hypo-perfusion by performing statistical neighbourhood-sensitive classification of voxels within synthetic parametric maps of perfusion indices. Statistically, the ischaemic lesion quantification is a process to label image pixels into different groups based on attributes like signal intensity and neighboring environments. This process can be implemented by some clustering algorithms. The Gaussian mixture model (GMM) is a traditional clustering model and the Expectation-Maximization(EM) algorithm can be applied for GMM training based on likelihood maximization. However, the ordinary Gaussian mixture model only takes the signal intensity into account. When the Signal-to-noise ratio (SNR) is poor, there might be some isolated "island" pixels labelled within other groups. Those "islands", in general, do not genuinely exist in DCE-MRI of patients with ischaemic 
heart disease because any specific heart wall segment is dominantly perfused by one main coronary artery. Any narrowing of that coronary will cause reduced blood supply to the whole heart wall segments. Isolated pixels whose intensities differ considerably from their neighbourhood can lead to spurious singleton clusters. This is physiologically unrealistic, i.e. due to physiological constraints we have prior knowledge that one large lesion is more likely than many isolated small lesions in myocardium [2]. Consequently, the spatial information should be taken into account in lesion quantification. For example, a pixel should be more likely labelled as "lesion" once its adjacent pixels are labelled as "lesion" too. To implement this idea, in this study, we will use the spatially variant finite mixture model (SVFMM) [3][4], which combines signal intensities with neighbourhood information, to improve the traditional Gaussian mixture model (GMM).

\section{The Gaussian Mixture Model}

Let $x^{i}$ denote the signal intensity at the $i^{\text {th }}$ pixel of an MR image $(i=1, \ldots, N)$, where $N$ is the total number of pixels in this image. The Gaussian Mixture Model (GMM) [5] assumes there are K clusters. In our case, we have $K=2$ clusters: "lesion tissue" and "healthy tissue". In the GMM, the parameters $\pi_{j}^{i}=P\left(j \mid x^{i}\right)$ denote the prior probabilities of the $i^{\text {th }}$ pixel belonging to the $j^{\text {th }}$ cluster $(j=1,2)$, and they have to satisfy the constrains that $0 \leq \pi_{j}^{i} \leq 1$ and $\pi_{1}^{i}+\pi_{2}^{i}=1$. Let $\pi_{j}$ denote the vector for cluster $j$, which is $\boldsymbol{\pi}_{j}=\left\{\pi_{j}^{1}, \pi_{j}^{2}, \ldots, \pi_{j}^{N}\right\}$, and let $\boldsymbol{\Pi}$ denote the set of probability vectors $\boldsymbol{\Pi}=\left\{\boldsymbol{\pi}_{\mathbf{1}}, \boldsymbol{\pi}_{\mathbf{2}}\right\}$. Similarly, $\boldsymbol{\Theta}=\left\{\boldsymbol{\theta}_{\mathbf{1}}, \boldsymbol{\theta}_{\mathbf{2}}\right\}$ denotes the set of component parameters, which are the parameters of the corresponding mixture components. The probability density function for each observation $x^{i}$ is

$$
f\left(x^{i} \mid \boldsymbol{\Pi}, \boldsymbol{\Theta}\right)=\pi_{1}^{i} \phi\left(x^{i} \mid \boldsymbol{\theta}_{\mathbf{1}}\right)+\pi_{2}^{i} \phi\left(x^{i} \mid \boldsymbol{\theta}_{\mathbf{2}}\right)
$$

where $\phi\left(x^{i} \mid \boldsymbol{\theta}_{\boldsymbol{j}}\right)$ is a Gaussian distribution with parameters $\boldsymbol{\theta}_{\boldsymbol{j}}=\left\{\mu_{j}, \sigma_{j}^{2}\right\}$. The parameter $\mu_{j}$ is the mean of the $j^{\text {th }}$ Gaussian component and $\sigma_{j}^{2}$ is its variance. Let $\mathbf{x}=\left\{x^{1}, x^{2}, \ldots, x^{N}\right\}$ denote the data, i.e. the set of all pixel intensities of the MR image. The log-likelihood function of the data is given by

$$
l(\boldsymbol{\Theta} \mid \mathbf{x}, \boldsymbol{\Pi})=\sum_{i=1}^{N} \log \left[\pi_{1}^{i} \phi\left(x^{i} \mid \boldsymbol{\theta}_{\mathbf{1}}\right)+\pi_{2}^{i} \phi\left(x^{i} \mid \boldsymbol{\theta}_{\mathbf{2}}\right)\right]
$$

The maximization of this so-called 'incomplete' log-likelihood function has no closed-form solution, due to the summation terms inside the logarithm. A standard procedure, therefore, is to use data augmentation and apply the EM algorithm [6]. To be specific, we include an unobserved binary latent variable $z_{j}^{i}[5]$, which indicates which component the $i^{\text {th }}$ pixel belongs to, and define the probability function

$$
f\left(x^{i}, z_{j}^{i} \mid \boldsymbol{\Pi}, \boldsymbol{\Theta}\right)=\left(\pi_{1}^{i} \phi\left(x^{i} \mid \boldsymbol{\theta}_{\mathbf{1}}\right)\right)^{z_{1}^{i}} \times\left(\pi_{2}^{i} \phi\left(x^{i} \mid \boldsymbol{\theta}_{\mathbf{2}}\right)\right)^{z_{2}^{i}}
$$

where the $z_{j}^{i}$ are subject to the constraints that $z_{j}^{i} \in\{0,1\}$ and $z_{1}^{i}+z_{2}^{i}=1$. This implies that any pixel $i$ can only be represented by one Gaussian component. Given the set of binary latent variables $z_{j}^{i}$, we have the complete log-likelihood function

$$
l(\boldsymbol{\Theta} \mid \mathbf{x}, \boldsymbol{\Pi}, \mathbf{Z})=\sum_{i=1}^{N}\left[z_{1}^{i}\left(\log \left(\pi_{1}^{i}\right)+\log \phi\left(x^{i} \mid \boldsymbol{\theta}_{\mathbf{1}}\right)\right)+z_{2}^{i}\left(\log \left(\pi_{2}^{i}\right)+\log \phi\left(x^{i} \mid \boldsymbol{\theta}_{\mathbf{2}}\right)\right)\right]
$$

where $\mathbf{Z}=\left\{\mathbf{z}_{\mathbf{1}}, \mathbf{z}_{\mathbf{2}}\right\}$ and $\mathbf{z}_{\mathbf{j}}=\left\{z_{j}^{1}, z_{j}^{2}, \ldots, z_{j}^{N}\right\}$. By substituting $z_{2}^{i}=1-z_{1}^{i}$ and $\pi_{2}^{i}=1-\pi_{1}^{i}$, we can maximize Eq. 4 4 for given latent variables $\mathbf{Z}$. To maximize the (incomplete) log likelihood in Eq.(2), we use the EM algorithm [6][5]. The first step 
(Expectation step or E-step) is to calculate the expected value of $z_{j}^{i}, \gamma_{j}^{i}$, which is

$$
\begin{aligned}
\gamma_{j}^{i}=E\left(z_{j}^{i} \mid x^{i}, \pi_{j}^{i}, \boldsymbol{\theta}_{\boldsymbol{j}}\right) & =\sum_{j=1}^{2} z_{j}^{i} f\left(z_{j}^{i} \mid x^{i}, \pi_{j}^{i}, \boldsymbol{\theta}_{\boldsymbol{j}}\right) \\
& =1 \times f\left(z_{j}^{i}=1 \mid x^{i}, \pi_{j}^{i}, \boldsymbol{\theta}_{\boldsymbol{j}}\right)+0 \times f\left(z_{j}^{i}=0 \mid x^{i}, \pi_{j}^{i}, \boldsymbol{\theta}_{\boldsymbol{j}}\right) \quad j=1,2 \\
& =\frac{\pi_{j}^{i} \phi\left(x^{i} \mid \boldsymbol{\theta}_{\boldsymbol{j}}\right)}{\pi_{1}^{i} \phi\left(x^{i} \mid \boldsymbol{\theta}_{\mathbf{1}}\right)+\left(1-\pi_{1}^{i}\right) \phi\left(x^{i} \mid \boldsymbol{\theta}_{\mathbf{2}}\right)} \quad j=1,2
\end{aligned}
$$

and substitute $z_{j}^{i}$ by $\gamma_{j}^{i}$ in Eq. (4), to give the expected complete log likelihood function. The next step (Maximization step or M-step) is to maximize this function with respect to the parameters $\left(\boldsymbol{\theta}_{1}, \boldsymbol{\theta}_{2}\right)$. This procedure is iterated until convergence, i.e. until a zero-gradient point of the (incomplete) log likelihood (2) is reached. Further details on the EM algorithm can be found in [6] and [5].

\section{The Spatially Variant Finite Mixture Model}

In the standard Gaussian mixture model, only the signal intensity is used, without considering any information from the spatial context. This drawback can be overcome by the spatially variant finite mixture model (SVFMM) [3][4]. In the SVFMM, a prior distribution over the parameter $\Pi[3][\overline{7}][\overline{8}]$ is introduced to take the spatial information into account, which is based on the following Gibbs function:

$$
P(\boldsymbol{\Pi})=\frac{1}{C} \exp (-U(\boldsymbol{\Pi}))
$$

where

$$
U(\boldsymbol{\Pi})=\beta \sum_{i=1}^{N} V_{\mathscr{N}_{i}}(\boldsymbol{\Pi})
$$

and $C$ is a normalizing constant to ensure the integral over $P(\boldsymbol{\Pi})$ is 1 . The so-called potential function $U(\boldsymbol{\Pi})$ depends on a regularization parameter $\beta>0$. Each term in the sum, $V_{\mathscr{N}_{i}}(\boldsymbol{\Pi})$, is a function of $\left\{\pi_{j}^{m}\right\}$ which contains the neighbourhood information of the $i^{\text {th }}$ pixel and is defined as

$$
V_{\mathscr{N}_{i}}(\boldsymbol{\Pi})=\sum_{m \in \mathscr{N}_{i}} g\left(u_{i, m}\right)
$$

where $\mathscr{N}_{i}$ is the set of neighbourhood pixels of the $i^{\text {th }}$ pixel (set to the 4 nearest neighbours in the present work) and $u_{i, m}$ is the distance between two mixing coefficients $\pi_{j}^{i}$ and $\pi_{j}^{m}$. Here we use the squared Euclidean distance

$$
u_{i, m}=\sum_{j=1}^{2}\left(\pi_{j}^{i}-\pi_{j}^{m}\right)^{2}
$$

The function $g(u)$ must be non-negative and monotonically increasing [3] [7] [8]. Standard choices are $g(u)=\left(1+u^{-1}\right)^{-1}[8]$ or $g(u)=u[3]$. For the work reported in the present paper, we have used $g(u)=u$.

\section{The EM Algorithm for the Spatially Variant Finite Mixture Model}

Similar to the general Gaussian mixture model, the EM algorithm is used for the spatially variant finite mixture model. In the Expectation step, the expected value of the latent variables $\mathbf{Z}$ is calculated as

$$
\gamma_{j}^{i}=E\left(z_{j}^{i} \mid x^{i}, \pi_{j}^{i}, \boldsymbol{\theta}_{\boldsymbol{j}}\right)=\operatorname{Pr}\left(z_{j}^{i}=1 \mid \boldsymbol{\theta}_{\boldsymbol{j}}\right)=\frac{\pi_{j}^{i} \phi\left(x^{i} \mid \boldsymbol{\theta}_{\boldsymbol{j}}\right)}{\pi_{1}^{i} \phi\left(x^{i} \mid \boldsymbol{\theta}_{\mathbf{1}}\right)+\left(1-\pi_{1}^{i}\right) \phi\left(x^{i} \mid \boldsymbol{\theta}_{\mathbf{2}}\right)} \quad j=1,2
$$


which is the same as it in the standard Gaussian mixture model. The Maximization step is different from the Gaussian mixture model. Instead of maximizing the expected complete log-likelihood function, the SVFMM method maximizes the expected posterior of $\boldsymbol{\Theta}$ and $\boldsymbol{\Pi}$, which can be computed by

$$
E[P(\boldsymbol{\Theta}, \boldsymbol{\Pi} \mid \mathbf{x})]=E\left[\frac{P(\mathbf{x} \mid \boldsymbol{\Theta}, \boldsymbol{\Pi}) P(\boldsymbol{\Pi})}{P(\mathbf{x})}\right] \propto E[P(\mathbf{x} \mid \boldsymbol{\Theta}, \boldsymbol{\Pi}) P(\boldsymbol{\Pi})]
$$

To maximize $E[P(\boldsymbol{\Theta}, \boldsymbol{\Pi} \mid \mathbf{x})]$, we can maximize the logarithm of $E[P(\mathbf{x} \mid \boldsymbol{\Theta}, \boldsymbol{\Pi}) P(\boldsymbol{\Pi})]$, which is

$$
Q_{\mathrm{MAP}}(\boldsymbol{\Pi}, \boldsymbol{\Theta})=\log \left(\prod_{i=1}^{N} f\left(x^{i}, \gamma_{j}^{i} \mid \boldsymbol{\Pi}, \boldsymbol{\Theta}\right) P(\boldsymbol{\Pi})\right) \propto \sum_{i=1}^{N} \sum_{j=1}^{2} \gamma_{j}^{i}\left[\log \left(\pi_{j}^{i}\right)+\log \left(\phi\left(x^{i} \mid \theta_{j}^{i}\right)\right)\right]-\beta \sum_{i=1}^{N} \sum_{m \in \mathscr{N}_{i}} g\left(u_{i, m}\right)
$$

We can maximize this function with respect to $\Pi$ and $\boldsymbol{\Theta}$ by calculating the corresponding partial derivatives and setting them to 0:

$$
\frac{\partial Q_{\mathrm{MAP}}}{\partial \mu_{j}}=\sum_{i=1}^{N} \gamma_{j}^{i} \times \frac{x^{i}-\mu_{1}}{2 \sigma_{j}^{2}}=0
$$

and

$$
\mu_{1}=\frac{\sum_{i=1}^{N} \gamma_{1}^{i} x^{i}}{\sum_{i=1}^{N} \gamma_{1}^{i}} \quad \text { and } \quad \mu_{2}=\frac{\left(N-\sum_{i=1}^{N} \gamma_{1}^{i}\right) x^{i}}{N-\sum_{i=1}^{N} \gamma_{1}^{i}}
$$

Similarly,

$$
\frac{\partial Q_{\mathrm{MAP}}}{\partial \sigma_{j}^{2}}=\sum_{i=1}^{N} \gamma_{1}^{i}\left(-\frac{1}{2 \sigma_{j}^{2}}+\frac{\left(x^{i}-\mu_{j}\right)^{2}}{2 \sigma_{j}^{2}}\right)=0
$$

and

$$
\sigma_{1}^{2}=\frac{\sum_{i=1}^{N} \gamma_{1}^{i}\left(x^{i}-\mu_{1}\right)^{2}}{\sum_{i=1}^{N} \gamma_{1}^{i}} \quad \text { and } \quad \sigma_{2}^{2}=\frac{\left(N-\sum_{i=1}^{N} \gamma_{1}^{i}\right)\left(x^{i}-\mu_{2}\right)^{2}}{N-\sum_{i=1}^{N} \gamma_{1}^{i}}
$$

For the parameters $\Pi$, we only need to consider $\pi_{1}^{i}$, as we can make use of the constraint $\pi_{2}^{i}=1-\pi_{1}^{i}$. Substituting $\pi_{2}^{i}=1-\pi_{1}^{i}$ and $\gamma_{2}^{i}=1-\gamma_{1}^{i}$ into Eq. 14 , we get

$$
Q_{\mathrm{MAP}}\left(\pi_{1}\right) \propto \gamma_{1}^{i} \log \pi_{1}^{i}+\left(1-\gamma_{1}^{i}\right) \log \left(1-\pi_{1}^{i}\right)-\beta \sum_{m \in \mathscr{N}_{i}} \sum_{j=1}^{2}\left(\pi_{j}^{i}-\pi_{j}^{m}\right)^{2}
$$

and

$$
\frac{\partial Q_{\mathrm{MAP}}}{\partial \pi_{1}^{i}}=\frac{\gamma_{1}^{i}}{\pi_{1}^{i}}-\frac{1-\gamma_{1}^{i}}{1-\pi_{1}^{i}}-4 \beta \sum_{m \in \mathscr{N}_{i}}\left(\pi_{1}^{i}-\pi_{1}^{m}\right)=0
$$

Since both $\pi_{1}^{i} \neq 0$ and $1-\pi_{1}^{i} \neq 0$, we multiply $\pi_{1}^{i}\left(1-\pi_{1}^{i}\right)$ on both sides to get

$$
h\left(\pi_{1}^{i}\right)=4 \beta M\left(\pi_{1}^{i}\right)^{3}-4 \beta\left(\sum_{m \in \mathscr{N}_{i}}\left[\pi_{1}^{m}+1\right]\right)\left(\pi_{1}^{i}\right)^{2}+\left[\left(4 \beta \sum_{m \in \mathscr{N}_{i}} \pi_{1}^{m}\right)-1\right] \pi_{1}^{i}+\gamma_{1}^{i}=0
$$

where $M$ is the size of the neighbourhood ( $M=4$ in the present work). The mixing parameters $\pi_{2}^{i}$ can be calculated by

$$
\pi_{2}^{i}=1-\pi_{1}^{i}
$$


By substituting $\pi_{1}^{i}=0$ and $\pi_{1}^{i}=1$, we have $h(0)=\gamma_{1}^{i}>0$ and $h(1)=1-\gamma_{1}^{i}<0$. Therefore, there must at least be one root located in the interval $(0,1)$, and this root is exactly what we want. In our study, we obtain the numerical solution of Eq. (21) by using a line search in the interval $(0,1)$. Note that for $\beta=0$, the SVFMM reduces to the standard GMM. This means that GMM is a special case of SVFMM.

Now, the EM algorithm for the SVFMM can be summarized as follows:

1. Initialize the parameters $\boldsymbol{\Pi}, \boldsymbol{\Theta}$. Define a fixed value for the regularization parameter $\beta$.

2. E step: evaluate $E\left(z_{j}^{i} \mid x^{i}, \pi_{j}^{i}, \boldsymbol{\theta}_{\boldsymbol{j}}\right)$ by Eq. 12 ,

3. M step: estimate $\mu_{1}, \mu_{2}, \sigma_{1}^{2}, \sigma_{2}^{2}, \pi_{1}^{i}, \pi_{2}^{i}$ given $E\left(z_{j}^{i} \mid x^{i}, \pi_{j}^{i}, \theta^{i}\right)$ by Eq. 16, Eq. 18, Eq. 21, and Eq. 22,

4. Evaluate $Q_{\mathrm{MAP}}$ using Eq. 14 ) and check for convergence. If the convergence criterion, i.e. $\left|Q_{\mathrm{MAP}}{ }_{\mathrm{OLD}}-Q_{\mathrm{MAP}}{ }_{\mathrm{NEw}}\right|<\xi$, where $\xi$ is a given positive small value, is not satisfied, return to step 2.

\section{Description of Clinical and Synthetic Data}

\subsection{Clinical data}

Our clinical data are derived from a BHF MR-MI clinical imaging cohort study (ClinicalTrials.gov, NCT02072850) involving patients with ST-segment elevation myocardial infarction (STEMI) [9]. A comprehensive cardiac MRI protocol included an assessment of resting perfusion using a T1-weighted, fast gradient echo DCE-MRI sequence, capturing three short axis slices within each heartbeat, before, during and immediately following intravenous administration of gadoterate meglumine (Gd2+-DOTA, Dotarem, Guebert S.A.). The DCE-MRI was acquired over approximately 1 minute.

The myocardial perfusion image we used in this paper is from one patient. There are 60 images taken from this patient during approximately 1 minute. The experimental image we used is taken at about the $50^{\text {th }}$ second and the signal intensity of this image is 1 dimension valued form 0 to 255 . The myocardium tissue in the experimental image is segmented by an MRI operator manually. There are 637 pixels located in the myocardium.

\subsection{Simulated data}

The synthetic data are designed so as to mimic the clinical data. The shape and the size of the synthetic myocardium is exactly the same as for the clinical data. The whole myocardium ring is divided into two parts. The top half is designed to be "healthy tissue" and the bottom half is designed to be "lesion tissue". The signal intensity of the "healthy tissue" group is sampled from $N\left(\mu_{h}, \sigma_{h}^{2}\right)$, and the signal intensity of the "lesion group" is sampled from $N\left(\mu_{l}, \sigma_{l}^{2}\right)$. The choice of parameters $\mu_{h}, \mu_{l}, \sigma_{h}^{2}, \sigma_{l}^{2}$ is empirical. To be specific, they are chosen according to the GMM results: $\mu_{h}$ and $\sigma_{h}^{2}$ have been set to the mean and variance of the "healthy tissue" groups from the GMM clustering results; similarly $\mu_{l}$ and $\sigma_{l}^{2}$ have been obtained from the "lesion tissue" group. The synthetic data is used to validate the algorithms. The details of the experiment results will be introduced in the next section.

This design of the synthetic data mimics the same SNR as for the clinical data. The number of wrongly segmented pixels using the GMM is positively correlated with the SNR value. Therefore, the performance of the GMM method for the synthetic data reflects the SNR for the clinical data.

\section{Experimental Results}

Fig.(1) shows the evolution of the log-posterior as a function of the number of EM cycles for different choices of the regularization parameter $\beta$. It can be seen that the rate of convergence depends on $\beta$, with increasing values of $\beta$ leading to a slower convergence.

Fig.(2) shows a comparison of the classification results 'healthy tissue' versus 'lesion' between GMM and SVFMM, for different values of $\beta$. The blue areas are labelled as "lesion tissue" and the yellow areas are labelled as "healthy tissue". The original MR image in the bottom right of panel (a) shows that there is a large shadow area located in the right bottom of the ring. However, the signal-to-noise ratio (SNR) is poor. Therefore, it is challenging to determine the lesion visually. Our 


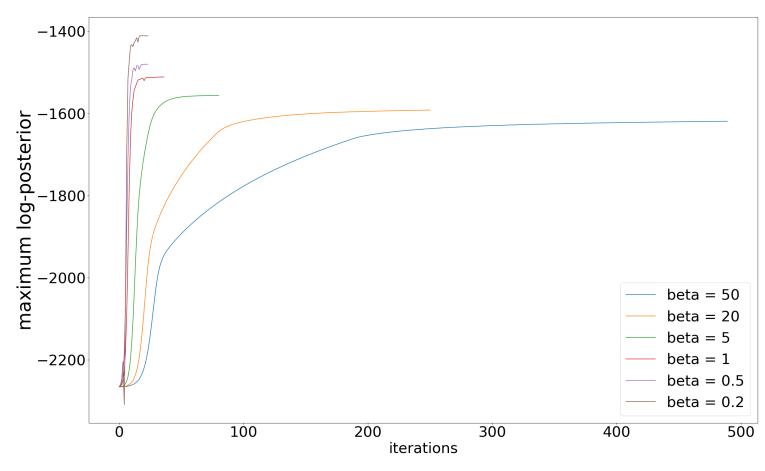

(a) clinical data

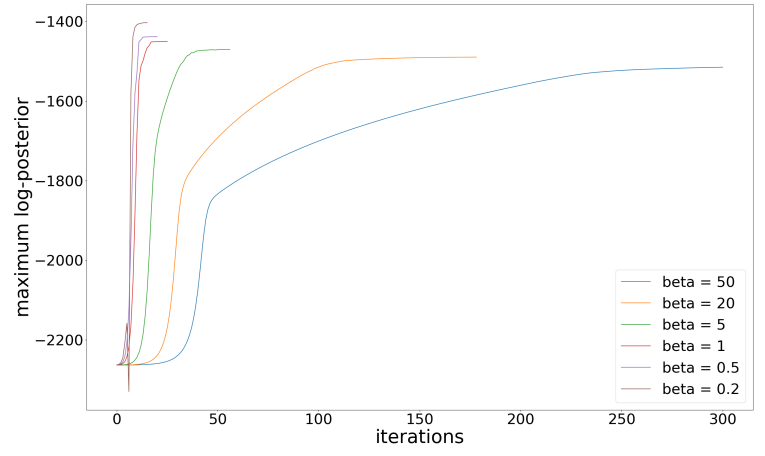

(b) synthetic data

Fig. 1: Evolution of the log-posterior for clinical (left panel) and synthetic (right panel) data during the optimization procedure. The horizontal axis shows the number of EM cycles.
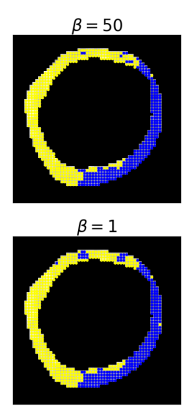

General GMM

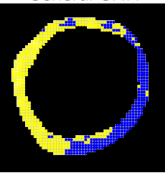

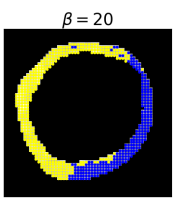

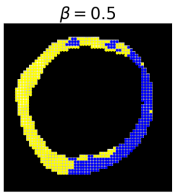

Original Myocardium

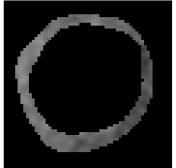

(a) clinical data
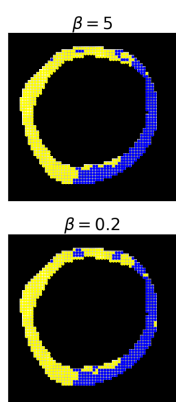

Original Image

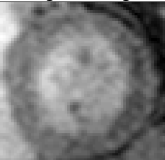

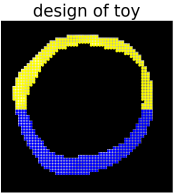
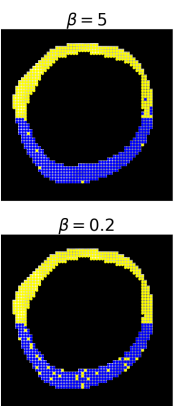
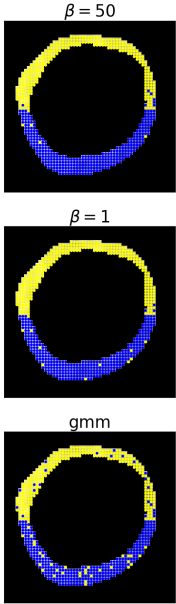
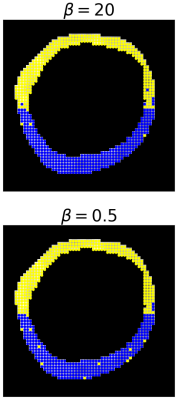

(b) synthetic data

Fig. 2: The comparison of lesion detection of clinical and synthetic data

These figures show the results of lesion detection with different methods and parameters. Panel (a) shows results for the clinical data, while panel (b) was obtained from the synthetic data. The blue areas are labelled as "lesion tissue" and the yellow areas are labelled as "healthy tissue". The centre bottom image in panel (a) is the segmented MRI myocardium grey map and the right bottom image in panel (a) is the original MRI left ventricle grey map. These two images are used to show the original source data.

results demonstrate that the segmentation obtained with the SVFMM is different from that obtained with the traditional GMM. There are fewer isolated pixels when applying the SVFMM method (for all values of $\beta$ ) to the clinical data. For the synthetic data, SVFMM leads to fewer misclassifications of pixels than GMM. This means that the SVFMM method indeed improves the segmentation in terms of the number of isolated pixels. Tables (1) and (2) summarize the numbers of isolated pixels and wrongly segmented (i.e. misclassified) pixels for both the clinical and the synthetic data. When $\beta=20$, the number of isolated and misclassified pixels is the lowest. The SVFMM has fewer than half the number of isolated pixels than the standard GMM for clinical data, and only about $\frac{1}{5}$ times the number of misclassified pixels than the standard GMM for synthetic data. 
Table 1: Total number of isolated pixels in the clinical data

\begin{tabular}{|c|c|}
\hline & total number of isolated pixels \\
\hline SVFMM $\beta=50$ & 27 \\
\hline SVFMM $\beta=20$ & 25 \\
\hline SVFMM $\beta=5$ & 32 \\
\hline SVFMM $\beta=1$ & 44 \\
\hline SVFMM $\beta=0.5$ & 47 \\
\hline SVFMM $\beta=0.2$ & 49 \\
\hline GMM & 59 \\
\hline
\end{tabular}

Table 2: Number of misclassified pixels in the synthetic data

\begin{tabular}{|c|c|}
\hline & total number of misclassified pixels \\
\hline SVFMM $\beta=50$ & 12 \\
\hline SVFMM $\beta=20$ & 10 \\
\hline SVFMM $\beta=5$ & 11 \\
\hline SVFMM $\beta=1$ & 14 \\
\hline SVFMM $\beta=0.5$ & 14 \\
\hline SVFMM $\beta=0.2$ & 33 \\
\hline GMM & 55 \\
\hline
\end{tabular}

The reason we use the number of isolated pixels as a performance criterion for the clinical data is that we do not know the real location or size of the lesion. However, according to the pathology of coronary heart diseases [2], it is more likely that there is only one large lesion than many isolated small lesions in the myocardium. The number of isolated pixels is hence used as a proxy criterion, due to the lack of a proper ground truth. For the synthetic data, on the other hand, we do have a proper gold standard, as we know the location and size of the lesion. We can therefore determine the number of misclassified pixels.

By taking the neighbourhood information into account, the total number of "island" pixels decreases substantially in the clinical data, and the number of misclassified pixels in the synthetic data also decreases dramatically. However, there are still some isolated pixels, and the performance on the synthetic data appears to be noticeably better than on the clinical data. There are two main reasons for this trend. Firstly, some pixels are located close to the endocardium and the epicardium (i.e. the two boundaries of the ring). In Fig.2(2)(a), in the centre bottom of the rings, some pixels are labelled as "healthy" but surrounded by "lesion". The reason is that these pixels are close to the blood pool. In DCE-MRI, the blood signal increases (bright, hyperintense in the grey-scale) and the zone of impaired perfusion appears dark (hypointense) due to a relative lack of contrast media mixed in blood. The hypointense zone spatially depicts the zone of impaired perfusion. Those pixels that are affected by blood will appear in the extreme bright range of the grey scale, and the segmentation will be affected accordingly. This effect has not been simulated in the synthetic data and constitutes the main reason why the SVFMM algorithm works better for the synthetic than the clinical data. Moreover, some isolated "islands" are located in some areas whose SNR (signal-to-noise ratio) is very low. A consequence of this low SNR value is that too many adjacent pixels get jointly misclassified, and the correction mechanism based on neighbourhood information, on which SVFMM is based, breaks down.

\section{Conclusion}

In the present paper, we have studied the problem of automatic lesion detection in dynamic contrast enhanced MRI scans of diseased myocardium, which reveal differences in microvascular perfusion. We have implemented the spatially variant finite mixture model (SVFMM) method, which is designed to take the neighborhood information of a pixel into account. This method has been compared with a traditional Gaussian mixture model (GMM) on both clinical and synthetic data. For the synthetic data, where the ground truth is known, we have directly quantified the classification performance. For the clinical data, we have used the isolated pixel count as a surrogate figure of merit. Both results suggest that SVFMM achieves 
significantly better results than the traditional GMM, suggesting that the inclusion of neighbourhood information substantially reduces the misclassification rate. However, even for the best results, there are still a substantial number of isolated and misclassified pixels, leaving room for future model improvement.

In our future work, we will apply methods for optimizing of the regularization parameter $\beta$, using nested cross-validation or Bayesian evidence optimization. More importantly, we will develop more realistic priors which take larger context information subject to discontinuities and the physiological particularities of how the blood flow affects the myocardial perfusion DCE-MRI scans into account.

\section{Acknowledgements}

This work was funded by the UK Engineering and Physical Sciences Research Council (EPSRC), grant number EP/N014642/1. Yalei Yang is funded by a grant from GlaxoSmithKline plc. Dirk Husmeier is supported by a grant from the Royal Society of Edinburgh, award number 62335. Colin Berry was supported by grants from the British Heart Foundation (PG/11/228474; $\mathrm{RE} / 18 / 6134217)$.

\section{References}

[1] J. A. Finegold, P. Asaria, and D. P. Francis, "Mortality from ischaemic heart disease by country, region, and age: statistics from world health organisation and united nations," International journal of cardiology, vol. 168, no. 2, pp. 934-945, 2013.

[2] M. Jerosch-Herold, "Quantification of myocardial perfusion by cardiovascular magnetic resonance," Journal of Cardiovascular Magnetic Resonance, vol. 12, no. 1, p. 57, 2010.

[3] S. Sanjay-Gopal and T. J. Hebert, "Bayesian pixel classification using spatially variant finite mixtures and the generalized EM algorithm," IEEE Transactions on Image Processing, vol. 7, no. 7, pp. 1014-1028, 1998.

[4] K. Blekas, A. Likas, N. P. Galatsanos, and I. E. Lagaris, "Mixture model based image segmentation with spatial constraints," in 2004 12th European Signal Processing Conference, pp. 2119-2122, IEEE, 2004.

[5] C. M. Bishop, Pattern recognition and machine learning. Springer, 2006.

[6] A. P. Dempster, N. M. Laird, and D. B. Rubin, "Maximum likelihood from incomplete data via the EM algorithm," Journal of the Royal Statistical Society: Series B (Methodological), vol. 39, no. 1, pp. 1-22, 1977.

[7] Y. Zhang, M. Brady, and S. Smith, "Segmentation of brain MR images through a hidden Markov random field model and the Expectation-Maximization algorithm," IEEE transactions on medical imaging, vol. 20, no. 1, pp. 45-57, 2001.

[8] P. J. Green, "Bayesian reconstructions from emission tomography data using a modified EM algorithm," IEEE transactions on medical imaging, vol. 9, no. 1, pp. 84-93, 1990.

[9] D. Carrick, C. Haig, S. Rauhalammi, N. Ahmed, I. Mordi, M. McEntegart, M. C. Petrie, H. Eteiba, S. Hood, S. Watkins, et al., "Prognostic significance of infarct core pathology revealed by quantitative non-contrast in comparison with contrast cardiac magnetic resonance imaging in reperfused st-elevation myocardial infarction survivors," European heart journal, vol. 37, no. 13, pp. 1044-1059, 2015. 